\title{
Kibble-Zurek scaling in quantum speed limits for shortcuts to adiabaticity
}

\author{
Ricardo Puebla $\odot,{ }^{1, *}$ Sebastian Deffner, ${ }^{2, \dagger}$ and Steve Campbell $\odot^{3, \sharp}$ \\ ${ }^{1}$ Centre for Theoretical Atomic, Molecular and Optical Physics, School of Mathematics and Physics, Queen's University Belfast, \\ Belfast BT7 1NN, United Kingdom \\ ${ }^{2}$ Department of Physics, University of Maryland, Baltimore County, Baltimore, Maryland 21250, USA \\ ${ }^{3}$ School of Physics, University College Dublin, Belfield Dublin 4, Ireland
}

(Received 15 June 2020; accepted 30 June 2020; published 20 July 2020)

\begin{abstract}
Geometric quantum speed limits quantify the tradeoff between the rate at which quantum states can change and the resources that are expended during the evolution. Counterdiabatic driving is a unique tool from shortcuts to adiabaticity to speed up quantum dynamics while completely suppressing nonequilibrium excitations. We show that the quantum speed limit for counterdiabatically driven systems undergoing quantum phase transitions fully encodes the Kibble-Zurek mechanism by correctly predicting the transition from adiabatic to impulse regimes. Our findings are demonstrated for three scenarios, namely the transverse field Ising model, the Landau-Zener model, and the Lipkin-Meshkov-Glick model.
\end{abstract}

DOI: 10.1103/PhysRevResearch.2.032020

A particularly promising approach to quantum computing relies on quantum annealing [1-4]. In this paradigm, which has been dubbed adiabatic quantum computing [5], a quantum system is initially prepared in the ground state of a wellcontrolled Hamiltonian $H_{\mathrm{i}}$. Then, the "computer" is allowed to evolve adiabatically—infinitely slowly— toward the ground state of the final Hamiltonian $H_{\mathrm{f}}$, which encodes the desired solution to the computation. Like all quantum information processing systems, adiabatic quantum devices are subject to the inevitable noise from the environment [6] and require quantum error correction [7]. However, in adiabatic quantum computing the situation is even more involved than in, e.g., the gate-based approach, since computational errors come in two different flavors [8]: (i) the "usual" errors that are due to the interaction with the environment [9] and control noise [10], and (ii) errors that originate in parasitic excitations of the finite-time driving of any realistic system. While powerful algorithms exist to mitigate the former [11,12], the latter are significantly harder to control.

At least from a bird's-eye view, so-called shortcuts to adiabaticity [13] seem exceptionally well-suited to address this issue [14-16]. A shortcut to adiabaticity is a finite-time, controlled process to obtain the final state that would result from infinitely slow, adiabatic driving. In particular, counterdiabatic driving $[17,18]$ is designed to keep the time-evolving quantum state on the adiabatic manifold at all times. However, with the exception of a few special scenarios $[19,20]$, the

\footnotetext{
*r.puebla@qub.ac.uk

$\dagger$ deffner@umbc.edu

`steve.campbell@ucd.ie
}

Published by the American Physical Society under the terms of the Creative Commons Attribution 4.0 International license. Further distribution of this work must maintain attribution to the author(s) and the published article's title, journal citation, and DOI. necessary control fields to facilitate the shortcut tend to be highly nonlocal, and thus of only limited practical use in many-body systems [21-25]. In addition, current formulations of shortcuts to adiabaticity are inadequate as a control technique for mitigating computational errors. Often, implementing any shortcut in lattice systems at least requires knowledge of the initial and final eigenspectrum, meaning that in order to correct for computational errors, the correct outcome has to be known [25], although recently there have been some efforts to mitigate this requirement through Floquet engineering [26].

Therefore, alternative strategies and phenomenological approaches, such as linear response theory [27,28], appear instrumental [29]. Within the realm of phenomenology, the Kibble-Zurek mechanism (KZM) [30,31] is arguably the most prominent approach to nonequilibrium quantum dynamics [32-42]. The quantum KZM can be understood as an extension of the quantum adiabatic theorem [43]. As long as the rate of driving is smaller than the energy gap between the ground state and the first excited state, barely any transitions occur and the dynamics remains essentially adiabatic. However, close to the critical point of a quantum phase transition (QPT), energy gaps close and excitations become inevitable. The "amount" of these excitations can then be predicted entirely from the rate at which the system is driven and from the critical exponents of the QPT [30,37]. A rather subtle issue relates to determining precisely when a quantum system transitions between the adiabatic and the impulse regimes $[33,44]$. As a rule of thumb, this transition is expected to occur when the "relaxation time," i.e., one over the energy gap, becomes identical to one over the driving rate [30]. However, the natural question arises whether this expectation can be made more precise.

Recently, it was shown that signatures of the KZM may be present in the quantum speed limit (QSL) [45] when implementing a shortcut to adiabaticity [46]. Thus, while counterdiabatic driving may not be adequate to mitigate all sources of computational errors, the control fields still contain 
essential information to develop phenomenological quantum error-correcting paradigms. Indeed, given that counterdiabatic driving perfectly cancels the excitations that would otherwise occur in the system due to arbitrary ramps, we ask the following question: Can we exploit what is learned from quantum control to quantitatively study the nonequilibrium dynamics we are suppressing? In the following, we answer in the affirmative, showing that quantum control provides a useful window through which nonequilibrium dynamics can be explored. We focus on three systems for which the control fields are analytically known, namely the transverse field Ising model (TFIM) $[21,22,32,36,47,48]$, the Landau-Zener (LZ) model [18], and the Lipkin-Meshkov-Glick (LMG) model $[23,49,50]$, and we examine the dynamics approaching and crossing the QPT.

Our results provide a means to quantify the range over which strategies to mitigate fundamentally noncorrectable errors are required, i.e., the length of the impulse regime. To this end, we bring together three distinct areas of research, namely shortcuts to adiabaticity, the QSL, and the KZM. We demonstrate that the QSL exhibits a behavior reminiscent of the adiabatic-impulse approximation with distinct minima occurring at the crossover between the regimes. Furthermore, their distance (between left and right of the critical point) is fully consistent with the prediction of the KZM, thus establishing that the speed of the controlled dynamics reveals details of the underlying universality class of the model.

Kibble-Zurek scaling in the quantum speed limit. We start by briefly reviewing the conceptual building blocks and by establishing notions and notations. Consider a time-dependent Hamiltonian $H_{0}(t)$ with instantaneous eigenvalues $\left\{\varepsilon_{n}(t)\right\}$ and eigenstates $\left\{\left|n_{t}\right\rangle\right\}$. An arbitrary evolution of an eigenstate will, in general, lead to nonadiabatic excitations being created. However, we can construct a Hamiltonian, $H(t)=H_{0}(t)+$ $H_{\mathrm{CD}}(t)$, such that the adiabatic solution of $H_{0}(t)$ is the exact solution of the dynamics generated by $H(t)$. Therefore, evolving according to this new Hamiltonian achieves effective adiabatic dynamics in a finite time. The counterdiabatic term (assuming units such that $\hbar=1$ ) is [13]

$$
H_{\mathrm{CD}}(t)=i\left[\partial_{t}\left|n_{t}\right\rangle\left\langle n_{t}|,| n_{t}\right\rangle\left\langle n_{t}\right|\right] .
$$

Implementing a controlled dynamics invariably incurs a thermodynamic toll for suppressing the nonequilibrium excitations, and there are several approaches to quantify this energetic cost [14,51-59]. A particularly useful measure proposed by Zheng et al. [60] relates the instantaneous cost to the norm of the counterdiabatic driving field [60,61],

$$
\left\|H_{\mathrm{CD}}(t)\right\| \propto \sqrt{\left\langle\partial_{t} n_{t} \mid \partial_{t} n_{t}\right\rangle} \equiv \partial_{t} C .
$$

Note that the proportionality constant is dictated by the specific choice of norm employed. For simplicity, we neglect this factor and work directly with what is essentially the geometric tensor.

Central to our analysis will be assessing the speed of the evolution. Within the framework of geometric quantum speed limits, a meaningful speed can be defined for any distance measure or metric [62]. For our purposes, we shall focus on the norm-based approach $[62,63]$, for which the QSL for the controlled dynamics is given by [46]

$$
\nu_{\mathrm{QSL}}(t)=\frac{\sqrt{\varepsilon_{n}^{2}(t)+\left(\partial_{t} C\right)^{2}}}{\cos \left(\mathcal{L}_{t}\right) \sin \left(\mathcal{L}_{t}\right)} .
$$

Here, $\mathcal{L}_{t}=\arccos \left|\left\langle\psi_{0} \mid \psi_{t}\right\rangle\right|$ denotes the Bures angle between initial and evolved state at time $t$ and $\partial_{t} C$ as given in Eq. (2). Equation (3) captures the tradeoff between the "bare" energetic change in the system and the additional resources necessary to achieve the controlled dynamics [46].

At this point, it is natural to question whether our choice of the version of QSL is crucial for the analysis. Over the past decade, a plethora of formulations has been proposed [45], where QSLs based on the quantum Fisher information $[64,65]$ give the tightest bound on the actual rate of change. However, such formulations are less useful for studying counterdiabatic driving as the speed along the geodesic of the quantum manifold [66] is agnostic to the specifics of the nonequilibrium dynamics. In the following, we will focus on the nonequilibrium excitations arising from driving systems through QPTs, and thus for our purposes only formulations of the QSL, such as in Eq. (3), that are sensitive to the full energy spectrum will do.

Quantum as well as classical phase transitions are characterized by the fact that close to the critical point both the correlation length, $\xi$, as well as the correlation time, $\tau$, diverge. Renormalization-group theory predicts $[47,67,68]$

$$
\xi(g)=\xi_{0}\left|g-g_{c}\right|^{-v} \quad \text { and } \tau(g)=\tau_{0}\left|g-g_{c}\right|^{-z v},
$$

where $g$ is a dimensionless parameter measuring the distance from the critical point $g_{c}, v$ is the spatial exponent, and $z$ is the dynamical critical exponent. Typically, in thermodynamic phase transitions $g$ denotes the relative temperature [69], whereas in QPTs $g$ is a relative external field [30,36]. As noted above, for slow-enough driving and far from the critical point, $\tau \ll t$, the dynamics of the system is essentially adiabatic. This means, in particular, that all nonequilibrium excitations and defects equilibrate much faster than they are created. Close to the critical point, $\tau \simeq t$, the situation changes dramatically, since the response "freezes out," and defects and excitations cannot "heal" any longer. If the external driving is linear, $g(t)=t / \tau_{q}$, and in the conventional phrasing of the KZM $[30,70]$, the transition from adiabatic to impulse regime is expected to happen when the rate of driving becomes equal to the rate of relaxation, or more formally at

$$
\hat{\tau}(\hat{t})=\hat{t} \quad \text { with } \quad \hat{\tau}=\left(\tau_{0} \tau_{q}^{z \nu}\right)^{1 /(z v+1)} .
$$

However, this rather hand-waving argument for where to situate the crossover cannot be considered entirely satisfactory. In particular, in unitary quantum dynamics, in which no direct "relaxation" can occur, a more rigorous treatment appears desirable.

We therefore propose that the size of the impulse regime is determined by the turning point of Eq. (3), and we identify $\hat{t}$ from the time when the speed is minimized, $t_{m}$, cf. Fig. 1(a). In critical systems with time dependent order parameter, $g(t)$, and where Eq. (1) is known, we find $\partial_{t} C \sim|\dot{\varepsilon} / \varepsilon|$ with $\varepsilon \sim$ $\left|g-g_{c}\right|^{z \nu}$ and $g_{c}$ is the critical point [71]. In the case of a linear ramp, $g(t)=t / \tau_{q}$ we immediately obtain

$$
v_{\mathrm{QSL}, \min } \sim \tau_{q}^{-z v /(1+z v)} \quad \text { and } t_{m} \sim \tau_{q}^{z v /(z v+1)}
$$



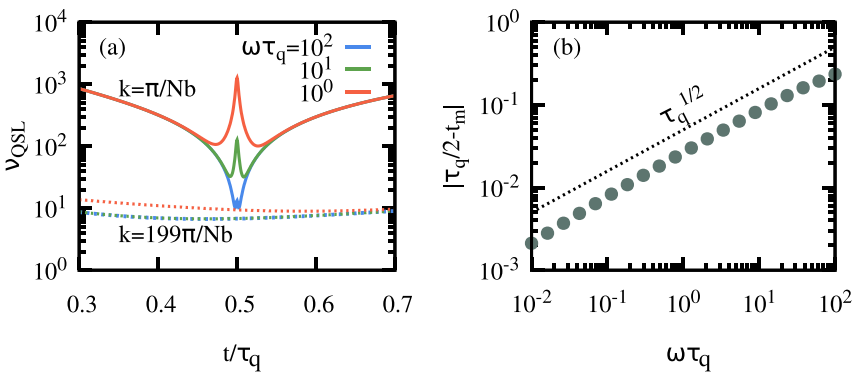

FIG. 1. (a) Speed $v_{\mathrm{QSL}}$ as given in Eq. (3) for the TFIM with counterdiabatic driving, quenched from $g(0)=0$ to $g\left(\tau_{q}\right)=2$ thus traversing the QPT and for different total ramp durations $\tau_{q}\left(\omega \tau_{q}=\right.$ $10^{2}, 10^{1}$, and $10^{\circ}$ ) and for two momenta $k=\pi / N b$ (solid lines) and $k=199 \pi / N b$ (dashed lines), with $N=1000$ spins. (b) Scaling of the duration of the impulse regime, i.e., of the time $\left|t_{c}-t_{m}\right|$, as a function of $\tau_{q}$, which shows KZM scaling $\tau_{q}^{z v /(1+z v)}=\tau_{q}^{1 / 2}$ (dashed line) for the TFIM as $z v=1$. Since $g\left(\tau_{q}\right)=2$, it follows that $t_{c}=$ $\tau_{q} / 2$. See the main text for further details.

which is precisely the scaling expected from the KZM. In the following we demonstrate this universal result, Eq. (6), for two many body systems at the opposite sides of the interaction spectrum - the transverse field Ising model and the LMGmodel.

One-dimensional transverse-field Ising model. We begin with the spin- $1 / 2$ nearest-neighbor Ising model in a transverse field $[47,48]$,

$$
H_{\text {TFIM }}(g)=-\omega \sum_{j=1}^{N}\left(g \sigma_{j}^{x}+\sigma_{j}^{z} \sigma_{j+1}^{z}\right),
$$

with $N$ even and, for convenience, we assume periodic boundary conditions, $\sigma_{N+1}^{x, y, z}=\sigma_{1}^{x, y, z}$, where $\sigma^{x, y, z}$ are the Pauli matrices. Upon the standard Jordan-Wigner and Fourier transformations, Eq. (7) decouples into a collection of independent Landau-Zener problems in momentum space $[32,47,48]$, $H_{\mathrm{TFIM}}(g)=\bigoplus_{k>0} \Psi_{k}^{\dagger} H_{k}(g) \Psi_{k}$, with $\Psi_{k}^{\dagger}=\left(c_{k}^{\dagger}, c_{-k}\right)$ the mode of Fourier-transformed fermionic operators and $H_{k}(g)=$ $h_{k}^{z}(g) \sigma_{z}^{k}+h_{k}^{x} \sigma_{x}^{k}$, where $\sigma_{x, y, z}^{k}$ are the Pauli matrices for momentum $k$, which takes discrete values $k_{n}=(2 n-1) \pi /(N b)$ with $n=1, \ldots, N / 2$, and $b$ is the distance between neighboring spins. From the transformation, one finds the coefficients $h_{k}^{z}(g)=2 \omega[g-\cos (k b)], h_{k}^{x}=2 \omega \sin (k b)$, and the groundstate energy for the $k$-momentum subspace reads (see [71] for details)

$$
\varepsilon_{k}(g)=-2 \omega \sqrt{g^{2}+1-2 g \cos (k b)}-2 \omega g .
$$

There is a QPT at $g_{c}=1$ [47], and for ramps that approach or cross the critical point in a finite time, $\tau_{q}$, we recover precisely the universal scaling laws from the predictions of the KZM [30,32,36]. Since the solution involves rewriting Eq. (7) into independent Landau-Zener models in momentum space, determining the associated counterdiabatic Hamiltonian, Eq. (1), becomes greatly simplified as it reduces to the concatenation of two-level controls $[18,21,22,46]$. We introduce the counterdiabatic driving, which for the $k$-subspace is $H_{k, \mathrm{CD}}[g(t)]=$ $\Theta_{k}[g(t)] \sigma_{y}^{k}$, with $\Theta_{k}(g)=h_{k}^{z}(g) h_{k}^{z}\left\{2\left[h_{k}^{z, 2}(g)+h_{k}^{x, 2}\right]\right\}^{-1}$. We can readily determine Eq. (2) for a given subspace, $\partial_{t} C=2\left|\Theta_{k}[g(t)]\right|$, and the Bures angle between ground states at $g_{0}$ and $g(t), \quad \mathcal{L}_{t}=\arccos \left|\left\langle\psi_{\mathrm{gs}}[g(t)] \mid \psi_{\mathrm{gs}}\left(g_{0}\right)\right\rangle\right|=$ $\arccos \left|\prod_{k>0} \cos \left(\theta_{k}[g(t)]-\theta_{k}\left(g_{0}\right)\right)\right|, \quad$ with $\quad \theta_{k}(g)=$ $\arctan \left(\left\{h_{k}^{z}(g)-\left[h_{k}^{z, 2}(g)+h_{k}^{x, 2}\right]^{1 / 2}\right\} / h_{k}^{x}\right)[71]$.

We now have all the ingredients necessary to evaluate the QSL, Eq. (3), and to begin we consider a linear ramp, $g(t)=$ $g_{f} t / \tau_{q}$. For low $k$ subspaces where $\varepsilon_{k}(g) \rightarrow 0$ for $g \rightarrow g_{c}$, and are therefore critical, the solid lines in Fig. 1(a) show that the speed exhibits a behavior reminiscent of the adiabatic-impulse approximation. Indeed, notice that all lines fall on top of each other far from the critical point. Thus, the quantum speed is independent of the ramp duration, indicating the model is in the adiabatic regime.

As the system approaches the critical point, and therefore crosses over into the impulse regime, we see quantitative differences emerge as we vary the ramp duration. Smaller $\tau_{q}$ leads to increased speeds in the vicinity of the QPT, while larger $\tau_{q}$ reduces the effective size of the critical region. This picture is consistent with the tradeoff between the speed and the energetic cost of implementing quantum control $[14,46,72-74]$ and demonstrates that, near the critical point, control protocols are essential for achieving finitetime adiabatic dynamics, while if the system is manipulated outside the impulse regime, where energy gaps are typically much larger, there is no need for complex control techniques.

With the qualitative picture established, we now inspect the scaling properties of the instant of the time, $t_{m}$, at which $v_{\text {QSL }}$ features a minimum. The intuition is clear: When no counterdiabatic control is necessary, the speed will be entirely dependent on the behavior of the ground-state energy; cf. Eq. (3). To adiabatically cross the QPT without any additional control, the speed must vanish at the critical point due to the closing energy gap. For finite-time ramps, as the system approaches the QPT defects will become increasingly more likely to be generated, and therefore the need for counterdiabatic control grows. For such a process, the transition from "effectively adiabatic" to "requires control" is reflected in the nonzero cost, which in turn leads to increased speeds near the QPT [46]. Therefore, the crossover from the adiabatic to impulse regimes is delineated by the minimum of $v_{\mathrm{QSL}}$, which takes place at a time instant $t_{m}$. We find that the duration of the impulse regime scales as $\left|t_{c}-t_{m}\right| \sim \tau^{1 / 2}$ with $t_{c}$ such that $g\left(t_{c}\right) \equiv g_{c}$, which is in perfect agreement with the expected scaling $\hat{\tau} \sim \tau^{z v /(1+z v)}=\tau_{q}^{1 / 2}$ given in Eq. (5) since $z v=1$ for the TFIM [30,32]. This is shown in Fig. 1(b), where fitting the points to a power law $\tau_{q}^{\beta}$ yields $\beta=0.51(1)$. Nonlinear protocols modify the KZM scaling in a nontrivial fashion. Nevertheless, we have confirmed that for $g(t)=1-(t-$ $\left.t / \tau_{q}\right)^{r}$ and $g(t) \propto\left(t / \tau_{q}\right)^{r}$ the speed of the controlled dynamics still exhibits excellent agreement with $\hat{\tau} \sim \tau_{q}^{z v r /(1+z v r)}$, as one expects from KZM arguments [71].

If we turn our attention to higher momentum subspaces, we find that the critical scaling is lost. In Fig. 1(a) the dashed lines correspond to a high momentum subspace with $k=199 \pi / N b$. Such high momentum subspaces do not show any trace of the QPT, and we find a trivial scaling with ramp duration $\propto \tau_{q}$ [71]. This is indeed expected since these subspaces are not critical, and hence KZM arguments do not 
apply. The energy gap remains large throughout the evolution, and as a result these subspaces do not contribute to the finitetime excitations in the bare nonequilibrium dynamics, making unnecessary the application of counterdiabatic control. This naturally emerges in our formalism: the trivial scaling in $v_{\mathrm{QSL}}$ in Eq. (3) accounts for the absence of criticality, i.e., for the absence of competing energy scales between the resources to achieve controlled dynamics and the energetic change in the considered subspace.

Finally, it is worth noting that KZM scaling was shown to be exhibited in the Landau-Zener problem in a seminal work by Damski [33], and therefore one may ask if we recover the same behavior in our setting. Due to its simplicity, we can fully analytically treat the Landau-Zener problem, and we find $t_{m} \sim \tau_{q}^{2 / 3}$ instead of the distinctive $\tau_{q}^{1 / 2}$ scaling for the TFIM [71]. In contrast to Ref. [33], no heuristic arguments are invoked here to determine the crossover between adiabatic and impulse regimes. Moreover, such a $\tau_{q}^{2 / 3}$ scaling is further supported from a numerical estimation of the crossover [71]. Since both the TFIM and the LZ exhibit the same KZM critical exponents, one naturally asks where the apparent discrepancy emerges. We find that the energy shift introduced when diagonalizing Eq. (7), i.e., the final term in Eq. (8), is crucial to recovering the predicted KZM scaling exponent in the true many-body case.

Lipkin-Meshkov-Glick model. As a final example, we move to a more complex many-body system. Originally introduced in the context of nuclear physics [49], the LMG model has become the paradigmatic system to study extreme long-range interactions and their role in critical phenomena, both theoretically [75-81] and experimentally [82-84]. The Hamiltonian can be written as

$$
H_{\mathrm{LMG}}(g)=-\omega J_{z}-\frac{g^{2} \omega}{N} J_{x}^{2},
$$

where $\omega$ sets the energy scale, while $g$ accounts for the relative strength of the ferromagnetic spin coupling, and $J_{\alpha}=\sum_{i=1}^{N} \sigma_{i}^{\alpha} / 2$ for $\alpha \in\{x, y, z\}$. In the thermodynamic limit, $N \rightarrow \infty$, the LMG can be diagonalized via a HolsteinPrimakoff transformation [71], which reveals a QPT at $g_{c}=1$ [75-78],

$$
H_{\mathrm{LMG}, \mathrm{eff}}(g)=\omega a^{\dagger} a-\frac{g^{2} \omega}{4}\left(a+a^{\dagger}\right)^{2},
$$

where $\left[a, a^{\dagger}\right]=1$ denote the bosonic excitations. The previous effective model is valid for $0 \leqslant g \leqslant 1$. Through an additional Bogoliubov transformation, Eq. (10) can be recast as a harmonic oscillator and therefore the corresponding counterdiabatic Hamiltonian is exactly known [23,85]. Note that $H_{\mathrm{LMG} \text {,eff }}(g)$ also corresponds to a low-energy effective description of other critical models [86-90].

We consider again a linear ramp through the QPT according to $g(t)=t / \tau_{q}$ for $t \in\left[0, \tau_{q}\right]$. The speed of the controlled evolution, as defined in Eq. (3), is given by [46,71]

$$
v_{\mathrm{QSL}}=\frac{\sqrt{\omega_{t}^{2} / 4+\left[\dot{\omega}_{t} /\left(\sqrt{8} \omega_{t}\right)\right]^{2}}}{\cos \mathcal{L}_{t} \sin \mathcal{L}_{t}}
$$
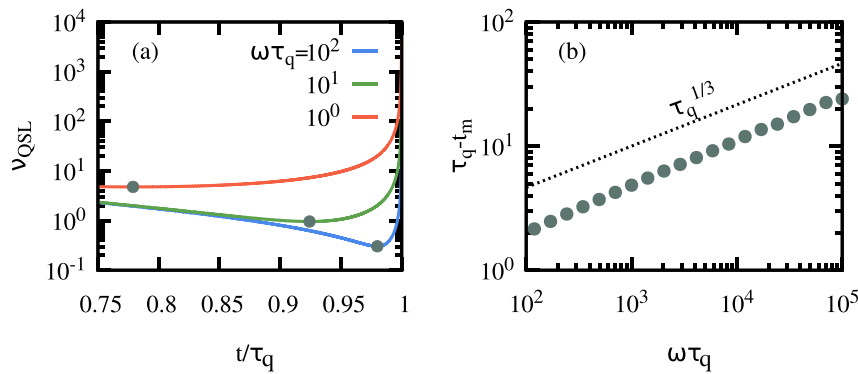

FIG. 2. (a) Speed $v_{\mathrm{QSL}}$ as given in Eq. (3) for the LMG model with counterdiabatic driving, quenched from $g(0)=0$ toward the QPT, $g\left(\tau_{q}\right)=g_{c}=1$, for different total times $\tau_{q}\left(\omega \tau_{q}=10^{2}, 10^{1}\right.$, and $\left.10^{\circ}\right)$. The minimum value of the speed, as well as its time location $t_{m} / \tau_{q}$, is indicated with solid points. (b) Scaling of the time $\left|t_{c}-t_{m}\right|$ with $t_{c}=\tau_{q}$ as $g\left(\tau_{q}\right)=g_{c}$, as a function of $\tau_{q}$, which shows the expected KZM scaling $\tau_{q}^{z \nu /(1+z v)}=\tau_{q}^{1 / 3}$ (dashed line) for the LMG as $z v=1 / 2$. See the main text for details.

with

$$
\begin{gathered}
\mathcal{L}_{t}=\arccos \left(\sqrt{2 \sqrt{\omega \omega_{t}} /\left(\omega+\omega_{t}\right)}\right) \\
\text { and } \omega_{t}=\omega \sqrt{1-g^{2}(t)} .
\end{gathered}
$$

In Fig. 2(a) we show the speed for different quench rates $\tau_{q}$. Similar to the TFIM, the rapid increase of $v_{\mathrm{QSL}}$ close to the QPT at $g=1$ suggests the presence of the two distinctive dynamical regimes predicted by the KZM. Computing the time, $t_{m}$, at which the speed features a minimum [cf. the solid points in Fig. 2(a)], we can analyze its scaling with the total quench time, $\tau_{q}$. From KZM arguments and for a linear ramp, one expects the uncontrolled dynamics, i.e., without counterdiabatic driving, to feature an impulse regime during a time $\hat{\tau} \sim \tau_{q}^{z v /(1+z \nu)}=\tau_{q}^{1 / 3}$ as $z v=1 / 2$ for the LMG universality class $[76,77,87,89]$. In Fig. 2(b) we confirm that the speed of the controlled dynamics exhibits the scaling $\left|\tau_{q}-t_{m}\right| \sim \tau_{q}^{1 / 3}$, which accounts for the duration of the impulse regime, precisely inline with the theoretical predictions. Indeed, a numerical fit to a power law $\tau_{q}^{\beta}$ in the interval $\omega \tau_{q} \in\left[10^{3}, 10^{5}\right]$ leads to $\beta=0.34(1)$.

Concluding remarks. Quantum error mitigation strategies are typically accompanied by a large computational or energetic overhead. The KZM provides a phenomenological framework to determine during which periods nonadiabatic excitations arise. Thus, in practice error mitigation needs only to be applied during these periods. In the present work, we have shown that this phenomenological prediction is encoded in the geometric QSL for counterdiabatic driving. Our findings crucially depend on the competition of the time-averaged energy of the bare Hamiltonian and the cost for counterdiabatic control. It is precisely this tradeoff that contains the signatures of the nonequilibrium behavior. Thus, it is not farfetched to realize that the paradigm of counterdiabatic driving may yet prove useful for adiabatic quantum computing. While complete control along the entire dynamics is generally unfeasible, the KZM demonstrates that only when the system is 
in the impulse regime are control fields needed. This period, which is the origin of all fundamentally noncorrectable errors, is indicated by the critical behavior of the QSL.

Acknowledgments. We acknowledge fruitful discussions with Adolfo del Campo. R.P. acknowledges the support by the SFI-DfE Investigator Programme (Grant No. 15/IA/2864).
This research was supported by Grant No. FQXi-RFP-1808 from the Foundational Questions Institute and Fetzer Franklin Fund, a donor advised fund of Silicon Valley Community Foundation (S.D.). S.C. gratefully acknowledges the Science Foundation Ireland Starting Investigator Research Grant "SpeedDemon" (No. 18/SIRG/5508) for financial support.
[1] T. Kadowaki and H. Nishimori, Quantum annealing in the transverse Ising model, Phys. Rev. E 58, 5355 (1998).

[2] R. Harris, M. W. Johnson, T. Lanting, A. J. Berkley, J. Johansson, P. Bunyk, E. Tolkacheva, E. Ladizinsky, N. Ladizinsky, T. Oh et al., Experimental investigation of an eightqubit unit cell in a superconducting optimization processor, Phys. Rev. B 82, 024511 (2010).

[3] M. W. Johnson, M. H. S. Amin, S. Gildert, T. Lanting, F. Hamze, N. Dickson, R. Harris, A. J. Berkley, J. Johansson, P. Bunyk et al., Quantum annealing with manufactured spins, Nature (London) 473, 194 (2011).

[4] S. Boixo, T. Albash, F. M. Spedalieri, N. Chancellor, and D. A. Lidar, Experimental signature of programmable quantum annealing, Nat. Commun. 4, 2067 (2013).

[5] T. Albash and D. A. Lidar, Adiabatic quantum computation, Rev. Mod. Phys. 90, 015002 (2018).

[6] B. C. Sanders, How to Build a Quantum Computer (IOP, Bristol, UK, 2017), pp. 2399-2891.

[7] J. Roffe, Quantum error correction: An introductory guide, Contemp. Phys. 60, 226 (2019).

[8] K. C. Young, M. Sarovar, and R. Blume-Kohout, Error Suppression and Error Correction in Adiabatic Quantum Computation: Techniques and Challenges, Phys. Rev. X 3, 041013 (2013).

[9] B. Gardas and S. Deffner, Quantum fluctuation theorem for error diagnostics in quantum annealers, Sci. Rep. 8, 17191 (2018).

[10] A. Wieckowski, S. Deffner, and B. Gardas, Disorder-assisted graph coloring on quantum annealers, Phys. Rev. A 100, 062304 (2019).

[11] K. L. Pudenz, T. Albash, and D. A. Lidar, Quantum annealing correction for random Ising problems, Phys. Rev. A 91, 042302 (2015).

[12] W. Vinci and D. A. Lidar, Scalable effective-temperature reduction for quantum annealers via nested quantum annealing correction, Phys. Rev. A 97, 022308 (2018).

[13] D. Guéry-Odelin, A. Ruschhaupt, A. Kiely, E. Torrontegui, S. Martínez-Garaot, and J. G. Muga, Shortcuts to adiabaticity: Concepts, methods, and applications, Rev. Mod. Phys. 91, 045001 (2019).

[14] A. C. Santos and M. S. Sarandy, Superadiabatic controlled evolutions and universal quantum computation, Sci. Rep. 5, 15775 (2015).

[15] K. Takahashi, Shortcuts to adiabaticity applied to nonequilibrium entropy production: an information geometry viewpoint, New J. Phys. 19, 115007 (2017).

[16] K. Takahashi, Hamiltonian engineering for adiabatic quantum computation: Lessons from shortcuts to adiabaticity, J. Phys. Soc. Jpn. 88, 061002 (2019).

[17] M. Demirplak and S. A. Rice, Adiabatic population transfer with control fields, J. Chem. Phys. A 107, 9937 (2003).
[18] M. Berry, Transitionless quantum driving, J. Phys. A 42, 365303 (2009).

[19] S. Deffner, C. Jarzynski, and A. del Campo, Classical and Quantum Shortcuts to Adiabaticity for Scale-Invariant Driving, Phys. Rev. X 4, 021013 (2014).

[20] C. Jarzynski, S. Deffner, A. Patra, and Y. Subaşı, Fast forward to the classical adiabatic invariant, Phys. Rev. E 95, 032122 (2017).

[21] A. del Campo, M. M. Rams, and W. H. Zurek, Assisted FiniteRate Adiabatic Passage Across a Quantum Critical Point: Exact Solution for the Quantum Ising Model, Phys. Rev. Lett. 109, 115703 (2012).

[22] B. Damski, Counterdiabatic driving of the quantum Ising model, J. Stat. Mech. (2014) P12019.

[23] S. Campbell, G. De Chiara, M. Paternostro, G. M. Palma, and R. Fazio, Shortcut to Adiabaticity in the Lipkin-Meshkov-Glick Model, Phys. Rev. Lett. 114, 177206 (2015).

[24] V. Mukherjee, S. Montangero, and R. Fazio, Local shortcut to adiabaticity for quantum many-body systems, Phys. Rev. A 93, 062108 (2016).

[25] S. Deffner and S. Campbell, Quantum Thermodynamics (Morgan \& Claypool, San Rafael, CA, 2019).

[26] P. W. Claeys, M. Pandey, D. Sels, and A. Polkovnikov, FloquetEngineering Counterdiabatic Protocols in Quantum ManyBody Systems, Phys. Rev. Lett. 123, 090602 (2019).

[27] T. V. Acconcia, M. V. S. Bonança, and S. Deffner, Shortcuts to adiabaticity from linear response theory, Phys. Rev. E 92, 042148 (2015).

[28] S. Deffner and M. V. S. Bonança, Thermodynamic control - an old paradigm with new applications, arXiv:2006.13761.

[29] M. Kolodrubetz, D. Sels, P. Mehta, and A. Polkovnikov, Geometry and non-adiabatic response in quantum and classical systems, Phys. Rep. 697, 1 (2017).

[30] W. H. Zurek, U. Dorner, and P. Zoller, Dynamics of a Quantum Phase Transition, Phys. Rev. Lett. 95, 105701 (2005).

[31] A. del Campo and W. H. Zurek, Universality of phase transition dynamics: Topological defects from symmetry breaking, Int. J. Mod. Phys. A 29, 1430018 (2014).

[32] J. Dziarmaga, Dynamics of a Quantum Phase Transition: Exact Solution of the Quantum Ising Model, Phys. Rev. Lett. 95, 245701 (2005).

[33] B. Damski, The Simplest Quantum Model Supporting the Kibble-Zurek Mechanism of Topological Defect Production: Landau-Zener Transitions from a New Perspective, Phys. Rev. Lett. 95, 035701 (2005).

[34] B. Damski and W. H. Zurek, Dynamics of a Quantum Phase Transition in a Ferromagnetic Bose-Einstein Condensate, Phys. Rev. Lett. 99, 130402 (2007).

[35] W. H. Dziarmaga, J. Zurek, and M. Zwolak, Non-local quantum superpositions of topological defects, Nat. Phys. 8, 49 (2012). 
[36] A. Francuz, J. Dziarmaga, B. Gardas, and W. H. Zurek, Space and time renormalization in phase transition dynamics, Phys. Rev. B 93, 075134 (2016).

[37] B. Gardas, J. Dziarmaga, and W. H. Zurek, Dynamics of the quantum phase transition in the one-dimensional Bose-Hubbard model: Excitations and correlations induced by a quench, Phys. Rev. B 95, 104306 (2017).

[38] B. Gardas, J. Dziarmaga, W. H. Zurek, and M. Zwolak, Defects in quantum computers, Sci. Rep. 8, 4539 (2018).

[39] A. del Campo, Universal Statistics of Topological Defects Formed in a Quantum Phase Transition, Phys. Rev. Lett. 121, 200601 (2018).

[40] F. J. Gómez-Ruiz and A. del Campo, Universal Dynamics of Inhomogeneous Quantum Phase Transitions: Suppressing Defect Formation, Phys. Rev. Lett. 122, 080604 (2019).

[41] M. M. Rams, J. Dziarmaga, and W. H. Zurek, Symmetry Breaking Bias and the Dynamics of a Quantum Phase Transition, Phys. Rev. Lett. 123, 130603 (2019).

[42] Z. Fei, N. Freitas, V. Cavina, H. T. Quan, and M. Esposito, Work Statistics Across a Quantum Phase Transition, Phys. Rev. Lett. 124, 170603 (2020).

[43] M. Born and V. Fock, Beweis des adiabatensatzes, Z. Phys. 51, 165 (1928).

[44] S. Deffner, Kibble-Zurek scaling of the irreversible entropy production, Phys. Rev. E 96, 052125 (2017).

[45] S. Deffner and S. Campbell, Quantum speed limits: from heisenberg's uncertainty principle to optimal quantum control, J. Phys. A 50, 453001 (2017).

[46] S. Campbell and S. Deffner, Trade-Off Between Speed and Cost in Shortcuts to Adiabaticity, Phys. Rev. Lett. 118, 100601 (2017).

[47] S. Sachdev, Quantum Phase Transitions, 2nd ed. (Cambridge University Press, Cambridge, 2011).

[48] A. Dutta, G. Aeppli, B. K. Chakrabarti, U. Divakaran, T. F. Rosenbaum, and D. Sen, Quantum Phase Transitions in Transverse Field Spin Models (Cambridge University Press, Cambridge, 2015).

[49] H. J. Lipkin, N. Meshkov, and A. J. Glick, Validity of manybody approximation methods for a solvable model, Nucl. Phys. 62, 188 (1965).

[50] S. Campbell, Criticality revealed through quench dynamics in the Lipkin-Meshkov-Glick model, Phys. Rev. B 94, 184403 (2016).

[51] X. Chen and J. G. Muga, Transient energy excitation in shortcuts to adiabaticity for the time-dependent harmonic oscillator, Phys. Rev. A 82, 053403 (2010).

[52] E. Torrontegui, I. Lizuain, S. González-Resines, A. Tobalina, A. Ruschhaupt, R. Kosloff, and J. G. Muga, Energy consumption for shortcuts to adiabaticity, Phys. Rev. A 96, 022133 (2017).

[53] A. Bravetti and D. Tapias, Thermodynamic cost for classical counterdiabatic driving, Phys. Rev. E 96, 052107 (2017).

[54] O. Abah and E. Lutz, Energy efficient quantum machines, Europhys. Lett. 118, 40005 (2017).

[55] A. Tobalina, J. Alonso, and J. G. Muga, Energy consumption for ion transport in a segmented paul trap, New J. Phys. 20, 065002 (2018).

[56] D. Sels and A. Polkovnikov, Minimizing irreversible losses in quantum systems by local counterdiabatic driving, Proc. Natl. Acad. Sci. USA 114, E3909 (2017).
[57] J. Li, T. Fogarty, S. Campbell, X. Chen, and T. Busch, An efficient nonlinear feshbach engine, New J. Phys. 20, 015005 (2018).

[58] B. Çakmak and Ö. E. Müstecaplıŏ̆lu, Spin quantum heat engines with shortcuts to adiabaticity, Phys. Rev. E 99, 032108 (2019).

[59] O. Abah, R. Puebla, A. Kiely, G. De Chiara, M. Paternostro, and S. Campbell, Energetic cost of quantum control protocols, New J. Phys. 21, 103048 (2019).

[60] Y. Zheng, S. Campbell, G. De Chiara, and D. Poletti, Cost of counterdiabatic driving and work output, Phys. Rev. A 94, 042132 (2016).

[61] M. Demirplak and S. A. Rice, On the consistency, extremal, and global properties of counterdiabatic fields, J. Chem. Phys. 129, 154111 (2008).

[62] S. Deffner, Geometric quantum speed limits: A case for Wigner phase space, New J. Phys. 19, 103018 (2017).

[63] S. Deffner and E. Lutz, Quantum Speed Limit for NonMarkovian Dynamics, Phys. Rev. Lett. 111, 010402 (2013).

[64] M. M. Taddei, B. M. Escher, L. Davidovich, and R. L. de Matos Filho, Quantum Speed Limit for Physical Processes, Phys. Rev. Lett. 110, 050402 (2013).

[65] A. del Campo, I. L. Egusquiza, M. B. Plenio, and S. F. Huelga, Quantum Speed Limits in Open System Dynamics, Phys. Rev. Lett. 110, 050403 (2013).

[66] I. Bengtsson and K. Życzkowski, Geometry of Quantum States: An Introduction to Quantum Entanglement (Cambridge University Press, Cambridge, 2017).

[67] M. E. Fisher, The renormalization group theory of critical behavior, Rev. Mod. Phys. 46, 597 (1974).

[68] I. Herbut, A Modern Approach to Critical Phenomena (Cambridge University Press, Cambridge, 2007).

[69] W. H. Zurek, Cosmological experiments in superfluid helium? Nature (London) 317, 505 (1985).

[70] W. H. Zurek, Cosmological experiments in condensed matter systems, Phys. Rep. 276, 177 (1996).

[71] See Supplemental Material at http://link.aps.org/supplemental/ 10.1103/PhysRevResearch.2.032020 for further explanations and details of the calculations including Refs. [32,46-49,7578].

[72] K. Funo, J.-N. Zhang, C. Chatou, K. Kim, M. Ueda, and A. del Campo, Universal Work Fluctuations During Shortcuts to Adiabaticity by Counterdiabatic Driving, Phys. Rev. Lett. 118, 100602 (2017).

[73] Z.-Y. Xu, W.-L. You, Y.-L. Dong, C. Zhang, and W. L. Yang, Generalized speed and cost rate in transitionless quantum driving, Phys. Rev. A 97, 032115 (2018).

[74] O. Lychkovskiy, O. Gamayun, and V. Cheianov, Time Scale for Adiabaticity Breakdown in Driven Many-Body Systems and Orthogonality Catastrophe, Phys. Rev. Lett. 119, 200401 (2017).

[75] P. Ribeiro, J. Vidal, and R. Mosseri, Exact spectrum of the Lipkin-Meshkov-Glick model in the thermodynamic limit and finite-size corrections, Phys. Rev. E 78, 021106 (2008).

[76] P. Ribeiro, J. Vidal, and R. Mosseri, Thermodynamical Limit of the Lipkin-Meshkov-Glick Model, Phys. Rev. Lett. 99, 050402 (2007)

[77] S. Dusuel and J. Vidal, Finite-Size Scaling Exponents of the Lipkin-Meshkov-Glick Model, Phys. Rev. Lett. 93, 237204 (2004). 
[78] S. Dusuel and J. Vidal, Continuous unitary transformations and finite-size scaling exponents in the Lipkin-Meshkov-Glick model, Phys. Rev. B 71, 224420 (2005).

[79] J. Vidal, S. Dusuel, and T. Barthel, Entanglement entropy in collective models, J. Stat. Mech. (2007) P01015.

[80] B. Žunkovič, M. Heyl, M. Knap, and A. Silva, Dynamical Quantum Phase Transitions in Spin Chains with Long-Range Interactions: Merging Different Concepts of Nonequilibrium Criticality, Phys. Rev. Lett. 120, 130601 (2018).

[81] T. Fogarty, S. Deffner, T. Busch, and S. Campbell, Orthogonality Catastrophe as a Consequence of the Quantum Speed Limit, Phys. Rev. Lett. 124, 110601 (2020).

[82] T. Zibold, E. Nicklas, C. Gross, and M. K. Oberthaler, Classical Bifurcation at the Transition from Rabi to Josephson Dynamics, Phys. Rev. Lett. 105, 204101 (2010).

[83] J. Zhang, G. Pagano, P. W. Hess, A. Kyprianidis, P. Becker, H. Kaplan, A. V. Gorshkov, Z.-X. Gong, and C. Monroe, Observation of a many-body dynamical phase transition with a 53-qubit quantum simulator, Nature (London) 551, 601 (2017).

[84] P. Jurcevic, H. Shen, P. Hauke, C. Maier, T. Brydges, C. Hempel, B. P. Lanyon, M. Heyl, R. Blatt, and C. F. Roos, Direct
Observation of Dynamical Quantum Phase Transitions in an Interacting Many-Body System, Phys. Rev. Lett. 119, 080501 (2017).

[85] J. G. Muga, X. Chen, S. Ibanez, I. Lizuain, and A. Ruschhaupt, Transitionless quantum drivings for the harmonic oscillator, J. Phys. B 43, 085509 (2010).

[86] R. Mottl, F. Brennecke, K. Baumann, R. Landig, T. Donner, and $\mathrm{T}$. Esslinger, Roton-type mode softening in a quantum gas with cavity-mediated long-range interactions, Science 336, 1570 (2012).

[87] M.-J. Hwang, R. Puebla, and M. B. Plenio, Quantum Phase Transition and Universal Dynamics in the Rabi Model, Phys. Rev. Lett. 115, 180404 (2015).

[88] R. Puebla, M.-J. Hwang, J. Casanova, and M. B. Plenio, Probing the Dynamics of a Superradiant Quantum Phase Transition with a Single Trapped Ion, Phys. Rev. Lett. 118, 073001 (2017).

[89] J. Peng, E. Rico, J. Zhong, E. Solano, and I. L. Egusquiza, Unified superradiant phase transitions, Phys. Rev. A 100, 063820 (2019).

[90] R. Puebla, A. Smirne, S. F. Huelga, and M. B. Plenio, Universal Anti-Kibble-Zurek Scaling in Fully Connected Systems, Phys. Rev. Lett. 124, 230602 (2020). 